\title{
Mineração
}

\section{Desenvolvimento sustentável e garimpo - 0 caso do Garimpo do Engenho Podre em Mariana, Minas Gerais}

\author{
(Sustainable development and garimpo - The case of the \\ Engenho Podre Garimpo in Mariana, Minas Gerais)
}

\author{
Pedro Amade \\ Universidade Federal de Ouro Preto - UFOP/PPGEM \\ E-mail:pedroamade@yahoo.com.br \\ Hernani Mota de Lima \\ Universidade Federal de Ouro Preto - UFOP/DEMIN \\ E-mail: hernani.lima@ufop.br
}

\section{Resumo}

Esse artigo avalia uma atividade garimpeira, garimpo de ouro do Engenho Podre, com base nos princípios de desenvolvimento sustentável. Esse estudo partiu da hipótese de que a implementação de um sistema de gestão ambiental, compromisso assumido pela Cooperativa dos Garimpeiros de Mariana (COOPERGAMA), na assinatura de um termo de ajuste de conduta, associado a uma melhora no desempenho técnico operacional, é possível, sendo que tal atividade pode ser levada adiante de forma sustentável com foco no tripé ambiental, econômico e social. Embora ainda marginal, do ponto de vista técnico, a COOPERGAMA adotou, durante o período de estudo, práticas ambientais e socio-econômicas em atendimento aos princípios de desenvolvimento sustentável. Entretanto aspectos administrativos, falta de investimentos e desorganização da cooperativa, entre outros fatores, ainda afetam a sustentabilidade de tais práticas a longo prazo. Esse artigo mostra que, uma vez adotadas as boas práticas ambientais e administrativas, é possível atender aos princípios de desenvolvimento sustentável numa atividade garimpeira.

Palavras-chave: Desenvolvimento sustentável, garimpo de ouro, meio ambiente.

\begin{abstract}
Based on sustainable development principles, this paper evaluates artisanal gold mining activity in Engenho Podre. This study followed the hypothesis that the implementation of environmental management according to a conduct adjustment agreement firmed by the Cooperativa dos Garimpeiros de Mariana (COOPERGAMA), associated with operational performance improvement would permit continuation of this activity in a sustainable way from the environmental, socioeconomic and cultural point of view. Although still illegal, from the technical point of view, the artisanal mining at Engenho Podre is adopting environmentally-accepted strategies to promote reasonable socioeconomic development. However, administrative issues and lack of investments still affect the sustainability of such practices in the long-term. This paper shows that when good administrative and environmental practices are adopted, it is possible to meet the sustainable development principles in an artisanal mining activity.
\end{abstract}

Keywords: Sustainable development, artisanal gold mining, Environment. 


\section{Desenvolvimento sustentável e mineração}

A definição de desenvolvimento sustentável é multidimensional. A primeira dimensão enfatiza a sustentabilidade do ambiente natural, que inclui a qualidade ambiental e o estoque dos recursos naturais. A segunda destaca a sustentabilidade econômica dos padrões de vida da sociedade. Segundo Eggert (2000), sustentabilidade econômica, no que diz respeito às atividades de mineração, envolve a transformação de capital mineral em capital humano. A ONU adota o Índice de Desenvolvimento Humano (IDH), que incorpora dados da renda bruta doméstica, do nível de educação, da expectativa de vida e de outros fatores relacionados com o desenvolvimento econômico. A terceira dimensão ressalta a sustentabilidade social e cultural. Mais difícil de medir, essa dimensão envolve o que é justo ou ético - conceitos sobre os quais há maior dificuldade de concordância entre as partes envolvidas. Eggert (2000) foca essa dimensão em duas questões principais - distribuição e processo. A distribuição dos benefícios e custos de uma mineração, por exemplo, pode não ser justa ou equitativa. Especificamente, os benefícios vão para os acionistas e para os governos (taxas) e, por outro lado, os custos associados são transferidos para a comunidade local na forma de rupturas sociais, riscos ambientais ou perda da identidade cultural. Processo, por outro lado, refere-se ao modo como decisões são tomadas e o papel das várias partes interessadas numa negociação. Nesse contexto, o processo de consulta e envolvimento das partes interessadas na tomada de decisão é fundamental para alcançar resultados socialmente e culturalmente sustentáveis.

Para promover o desenvolvimento sustentável, as empresas de mineração devem integrar as dimensões econômica, social e ambiental a suas atividades (Mikesell, 1994). Precisam mudar o foco de uma resposta ambiental end-of-pipe para um tratamento mais socialmente responsável. Devem forjar uma parceria com a comunidade com base no reconhecimento do potencial produtivo, social e cultural da comunidade, buscando uma melhoria do padrão de vida e de renda da comunidade envolvida, criando meios para a preservação de seus valores sociais e culturais (Epps \& Brett, 2000). Finalmente, visto que as minas não duram para sempre, empresas de mineração têm sido orientadas em usar sua capacidade técnica/financeira para estimular governos locais no desenvolvimento de novos negócios não ligados à mineração (Khanna, 2000). Tal atitude, certamente, poderá propiciar aumento nos serviços de infraestrutura e maior ocupação da mão-de-obra existente, reduzindo a pressão econômica e social sobre a mina.

Cragg (1998) defende a adoção dos princípios de desenvolvimento sustentável no planejamento, operação e fechamento de uma mina como forma de tornar a atividade defensável e acreditável a longo prazo. James (2000), por sua vez, ressalta que, para a mineração atender aos princípios do desenvolvimento sustentável, seus negócios devem ser tratados com foco no tripé - preservação ambiental, crescimento econômico e social - dentro de sua estratégia de negócios.

A difusão dos princípios de desenvolvimento sustentável trouxe três grandes conseqüências para a mineração. Primeiro, levou à inclusão dos aspectos culturais, econômicos e sociais de um projeto de mineração para as comunidades locais, desde a fase de exploração, até o pós-fechamento de uma mina (Clark \& Clark, 1996; Miller, 1997; Munchenberg, 1998). Atualmente, a consideração ambiental envolve não somente a preservação de um ecossistema e a garantia de segurança da comunidade. $\mathrm{O}$ foco mudou para uma visão mais ampla do bem-estar humano e dos direitos dos habitantes locais, passando a se preocupar com a qualidade de suas vidas e com as gerações futuras. Segundo, os princípios de desenvolvimento sustentável demandam a consideração de crescimento econômico e preservação ambiental desde o início de um projeto proposto. Terceiro, incluem a avaliação, em nível de projeto, dos valores morais e de ética, bem como levando em consideração valores subjetivos da comunidade, ao invés de apenas, enfatizar o tradicional valor econômico.

Os elementos anteriormente listados são intrinsecamente ligados e sugerem que o desenvolvimento sustentável seja avaliado numa escala mais ampla do que no nível de uma mina individual. A mineração deve, portanto, maximizar sua contribuição ao desenvolvimento sustentável numa escala maior. Em termos práticos, significa minimizar os impactos ambientais e maximizar os ganhos econômicos e sociais obtidos da explotação de um recurso mineral. A questão crítica está em se atingir um equilíbrio sustentável. Simplesmente maximizar os retornos econômicos e sociais não contribuirá para o desenvolvimento sustentável. O progresso nestes três itens varia consideravelmente, especialmente na questão social, ainda pouco desenvolvida, como exemplificado por Warhurst, Macfarlane al. (1999).

\section{Estudo de caso}

O garimpo do Engenho Podre é operado pela Cooperativa Regional Garimpeira de Mariana - COOPERGAMA, criada em 17/04/2004 para extração de ouro por draga no Rio Gualaxo do Norte (Figuras 1). Em 2005, após paralisação de suas atividades pela FEAM, a COOPERGAMA conseguiu reabrir o garimpo através de um Termo de Ajuste de Conduta (TAC), o qual envolveu a FEAM, o IGAM, o DNPM e o Ministério Público. O TAC incluía, entre outras medidas a serem tomados pela COOPERGAMA, a elaboração e apresentação do Relatório de Pesquisa e do Plano de Aproveitamento Econômico (PAE) ao DNPM e dos Planos de Reabilitação de Degradadas (PRAD), de Controle Ambiental (PCA) e Relatório de Controle Ambiental (RCA) à FEAM. Em adição aos consultores contratados para elaboração dos referidos planos, a COOPERGAMA foi também assistida pelo Departamento de Engenharia da Minas da Universidade Federal de Ouro Preto, via projetos de pesquisa financiados pela Fundação de Amparo à Pesquisa do Estado de Minas 
Gerais (FAPEMIG), com base nos editais de demanda induzida para formalização de Aglomerados Produtivos Locais (APL's).

A atividade garimpeira na região existe desde a chegada dos Bandeirantes há mais de 300 anos. Intensa entre 1700 e 1750 , pode-se afirmar que, desde então, o garimpo tem demonstrado sustentabilidade enquanto atividade importante para a economia e cultura da região. Embora historicamente minimizador de pressão social, o garimpo, dado ao conjunto de práticas administrativas e ambientais inadequadas, está distante de atender aos princípios de desenvolvimento sustentável. Desse modo, o presente estudo buscou avaliar a contribuição do garimpo de ouro para o desenvolvimento sócio-econômico e cultural de Monsenhor Horta (Mariana - MG) e suas implicações ambientais e tecer as relações entre Desenvolvimento Sustentável e a atividade garimpeira.

\section{Metodologia}

O presente trabalho consistiu em duas etapas. Uma envolveu o levantamento de dados sócio-econômicos do Garimpo do Engenho Podre, por meio de entrevistas com garimpeiros, cooperados (proprietários das dragas), visitas à Pre- feitura de Mariana para coleta de informações sobre o Distrito de Monsenhor Horta, de forma a permitir uma avaliação da importância do garimpo no Distrito e seu impacto econômico e social. A outra consistiu na análise dos procedimentos operacionais e de gestão do garimpo e suas implicações no aproveitamento do recurso mineral e no meio ambiente.

\section{Resultados e discussão}

\subsection{Diagnóstico sócio- econômico}

Uma análise dos dados coletados no garimpo demonstrou:

Baixa faixa etária dos garimpeiros. A idade média de 25 anos, inferior à média nacional (33 anos), segundo BARRETO (2000). Os garimpeiros de Monsenhor Horta começam mais cedo que os do quadro nacional. A proximidade do garimpo do Distrito de Monsenhor Horta e a atratividade do ganho fácil que tal atividade desperta nos jovens do local podem se constituir na razão pelo referido fato. A outra é cultural - a atividade garimpeira faz parte da cultura local há mais de 300 anos (75\% dos garimpeiros do Engenho Podre são de gerações de garimpeiros).
Baixo grau de escolaridade. Os analfabetos representam $23 \%$ do total de garimpeiros, sendo os demais distribuídos em primário (62\%), médio (14\%) e superior incompleto (1\%). Embora Monsenhor Horta também possua ensino médio noturno, a baixa escolaridade dos garimpeiros é justificada pela dificuldade de acesso à escola (os garimpeiros passam a semana no garimpo, distante $30 \mathrm{~km}$ do Distrito). A baixa escolaridade, por outro lado, pode justificar a atração pelo garimpo. De um modo geral, o garimpo não requer mão-de-obra qualificada, tampouco melhor nível educacional. Mais ainda, a grande oferta de vagas geradas nas mineradoras da região tem pouca influência no garimpo, visto ser a exigência de segundo grau completo para tais vagas um ponto de corte considerável.

Renda média mensal alta dos garimpeiros. No período do estudo, a COOPERGAMA possuía 22 cooperados, que operavam 26 dragas no rio Gualaxo do Norte, com cinco a seis funcionários em cada, os quais recebem $5 \%$ da produção bruta de ouro. A produção da COOPERGAMA contabilizada de 15 de março a 15 de novembro de 2006 é apresentada na Tabela 1. A produção média mensal do Garimpo do Engenho Podre cor-

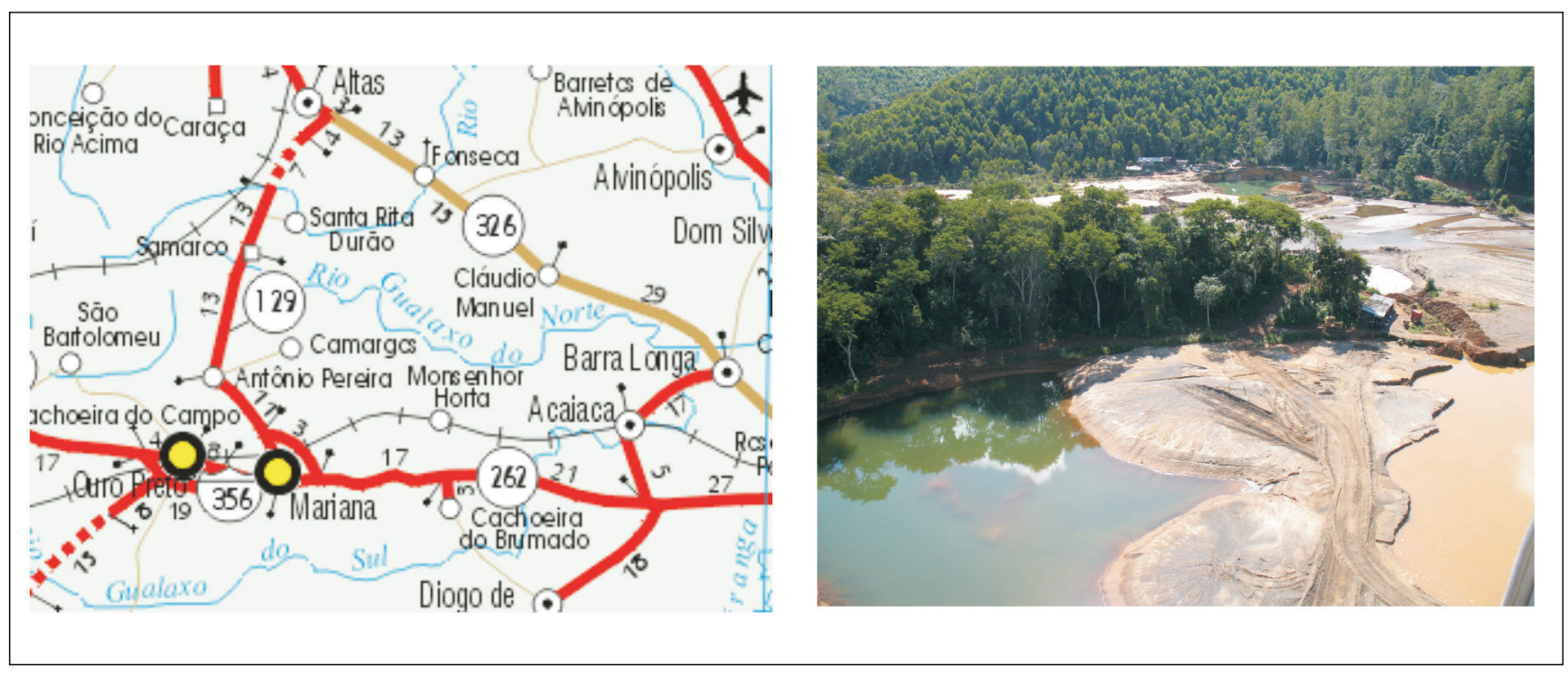

Figura 1 - Mapa de localização do Garimpo do Engenho Podre (esquerda) e vista aérea do garimpo (direita). 
Desenvolvimento sustentável e garimpo - O caso do Garimpo do Engenho Podre em Mariana, Minas Gerais

respondeu a 9,1 kg de Au. Considerouse, para os cálculos da receita gerada, o valor do grama de ouro em $\mathrm{R} \$ 40,00$ (valor de venda no garimpo). Dessa forma, pode-se chegar a uma renda líquida média de $\mathrm{R} \$ 624,28$ por garimpeiro por mês. Os garimpeiros pesquisados têm em mé- dia três anos na atividade, com um turno de trabalho de 8 horas e 5,5 dias de serviço por semana. Aproximadamente, $85 \%$ dos garimpeiros são responsáveis pela renda familiar e corresponde a $26 \%$ da população do distrito de Monsenhor Horta.
Pode-se, também, observar a participação da COOPERGAMA em projetos sociais via doação financeira para construção da igreja na localidade de Ponte do Gama, manutenção da ambulância do distrito, ajuda à banda local nas festividades de comemoração dos 170 anos de sua fundação e apoio aos eventos religiosos.

Tabela 1 - Resultados da Produção de ouro no garimpo do Engenho Podre.

\section{Dados Econômicos (em Reais)}

\begin{tabular}{|c|c|c|c|c|c|c|c|}
\hline Draga & $\begin{array}{l}\text { Produção } \\
\text { (gramas) }\end{array}$ & $\begin{array}{c}\text { Coopergama } \\
5 \%\end{array}$ & $\begin{array}{c}\text { Custo } \\
35 \%\end{array}$ & $\begin{array}{c}\text { Pessoal } \\
30 \%\end{array}$ & $\begin{array}{c}\text { Lucro } \\
30 \%\end{array}$ & $\begin{array}{c}\text { Mensal / } \\
\text { Garimpeiro }\end{array}$ & Garimpeiros \\
\hline$A$ & $3.230,70$ & $6.461,40$ & $45.229,80$ & $38.768,40$ & $38.768,40$ & 807,68 & 6 \\
\hline $\mathrm{B}$ & $4.328,00$ & $8.656,00$ & $60.592,00$ & $51.936,00$ & $51.936,00$ & $1.082,00$ & 6 \\
\hline $\mathrm{C}$ & $2.613,70$ & $5.227,40$ & $36.591,80$ & $31.364,40$ & $31.364,40$ & 653,43 & 6 \\
\hline $\mathrm{D}$ & 515,30 & $1.030,60$ & $7.214,20$ & $6.183,60$ & $6.183,60$ & 128,83 & 6 \\
\hline E & $4.128,30$ & $8.256,60$ & $57.796,20$ & $49.539,60$ & $49.539,60$ & $1.032,08$ & 6 \\
\hline $\mathrm{F}$ & $1.663,80$ & $3.327,60$ & $23.293,20$ & $19.965,60$ & $19.965,60$ & 415,95 & 6 \\
\hline$G$ & $11.820,00$ & $23.640,00$ & $165.480,00$ & $141.840,00$ & $141.840,00$ & 985,00 & 18 \\
\hline $\mathrm{H}$ & $2.068,30$ & $4.136,60$ & $28.956,20$ & $24.819,60$ & $24.819,60$ & 517,08 & 6 \\
\hline I & $7.252,90$ & $14.505,80$ & $101.540,60$ & $87.034,80$ & $87.034,80$ & 604,41 & 18 \\
\hline$J$ & $1.042,90$ & $2.085,80$ & $14.600,60$ & $12.514,80$ & $12.514,80$ & 260,73 & 6 \\
\hline K & $1.582,00$ & $3.164,00$ & $22.148,00$ & $18.984,00$ & $18.984,00$ & 395,50 & 6 \\
\hline $\mathrm{L}$ & $2.101,90$ & $4.203,80$ & $29.426,60$ & $25.222,80$ & $25.222,80$ & 525,48 & 6 \\
\hline M & $3.036,50$ & $6.073,00$ & $42.511,00$ & $36.438,00$ & $36.438,00$ & 759,13 & 6 \\
\hline $\mathrm{N}$ & 690,70 & $1.381,40$ & $9.669,80$ & $8.288,40$ & $8.288,40$ & 172,68 & 6 \\
\hline 0 & $4.674,20$ & $9.348,40$ & $65.438,80$ & $56.090,40$ & $56.090,40$ & 779,03 & 9 \\
\hline$P$ & 920,70 & $1.841,40$ & $12.889,80$ & $11.048,40$ & $11.048,40$ & 230,18 & 6 \\
\hline$Q$ & 354,90 & 709,80 & $4.968,60$ & $4.258,80$ & $4.258,80$ & 88,73 & 6 \\
\hline $\mathrm{R}$ & $2.434,40$ & $4.868,80$ & $34.081,60$ & $29.212,80$ & $29.212,80$ & 608,60 & 6 \\
\hline$S$ & $2.353,00$ & $4.706,00$ & $32.942,00$ & $28.236,00$ & $28.236,00$ & 392,17 & 9 \\
\hline $\mathrm{T}$ & $16.375,70$ & $32.751,40$ & $229.259,80$ & $196.508,40$ & $196.508,40$ & $2.046,96$ & 12 \\
\hline Total & $73.187,90$ & $146.375,80$ & $1.024 .630,60$ & $878.254,80$ & $878.254,80$ & & 156 \\
\hline Mensal & $9.148,49$ & $18.296,98$ & $128.078,83$ & $109.781,85$ & $109.781,85$ & 624,28 & \\
\hline
\end{tabular}




\subsection{Diagnóstico operacional da atividade garimpeira}

O processo de extração de ouro adotado no Garimpo do Engenho Podre é rudimentar e predatório, com perdas consideráveis na recuperação do ouro, decorrentes da ausência de uma pesquisa mineral sistemática, e da falta de planejamento de extração e do uso de equipamentos rudimentares e mal dimensionados (Figura 2). Estudos conduzidos no Departamento de Engenharia de Minas da UFOP demonstraram que a recuperação de ouro no garimpo varia entre 30 a 35\%. Razões para a baixa recuperação incluem a utilização de equipamentos rudimentares (sluices), o baixo nível educacional dos garimpeiros, o amadorismo administrativo com implicações na disponibilidade de peças para reparo, manutenção e reposição de motores e bombas e a falta de suporte técnico. Esses aspectos implicam uma utilização predatória e não sustentável, do ponto de vista técnico e econômico da reserva mineral.

\subsection{Diagnóstico ambiental da garimpagem no rio Gualaxo do Norte}

O diagnóstico ambiental possibilitou concluir que, em todo o processo de extração, separação, concentração, amal- gamação e queima do amálgama, há inúmeros impactos ambientais e consideráveis riscos à segurança e à saúde dos garimpeiros. Os impactos ambientais incluem a supressão da mata ciliar, assoreamento do rio Gualaxo do Norte e aumento da turbidez decorrente do lançamento do rejeito das calhas diretamente no leito do rio, vazamento de óleo e graxas, perda de mercúrio na amalgamação e queima do amálgama ao ar livre sem utilização de retortas. A almagamação e a queima do amálgama, em adição à ausência de condições higiênicas adequadas (instalações sanitárias, bebedouros etc.), além da presença de alojamentos insalubres constituem os itens de riscos à saúde e segurança dos garimpeiros.

Mudanças no processo produtivo foram executadas durante a vigência dos projetos de pesquisa apoiados pela FAPEMIG e geraram resultados positivos, tanto na produção, como na minimização dos impactos ambientais e na redução dos riscos à saúde e à segurança. Entre essas mudanças, destacam-se a criação de um laboratório para amalgamação, a coleta seletiva de lixo, a instalação de caixas com espuma para captação de óleos e graxas, que vazavam dos motores e reservatórios e o lançamento dos rejeitos dos sluices em catas desativadas.

Tais mudanças, cujos objetivos foram amenizar os impactos ambientais e atender às solicitações constantes no $\mathrm{TAC}$, foram plenamente aprovadas e adotadas no garimpo. A construção do laboratório de amalgamação buscou atender a duas solicitações. Uma ambiental, que visava a minimizar o lançamento de mercúrio nas margens do rio, e a outra visava a concentrar a produção em determinado local, de forma a melhorar o controle da produção por parte da COOPERGAMA e maximizar o recolhimento do percentual da cooperativa. Uma secretária foi contratada para esse trabalho.

As demais mudanças tiveram foco na minimização dos impactos ambientais da atividade. Vistorias ambientais posteriores, executadas pela FEAM, comprovaram o sucesso dessas medidas.

Uma alteração no processo produtivo, entretanto, faz-se necessária para aumentar a recuperação do ouro. No entendimento dos pesquisadores envolvidos, o que realmente ocorre no garimpo é uma mudança do ouro de local, uma vez que os sluices apresentavam baixa recuperação. Entretanto modificações no processo envolvem recursos financeiros para aquisição de equipamentos tecnologicamente mais adequados e treinamento dos garimpeiros. Outro projeto submetido e aprovado pela FAPEMIG, que inclui a substituição dos equipamentos, está sendo adotado no garimpo.

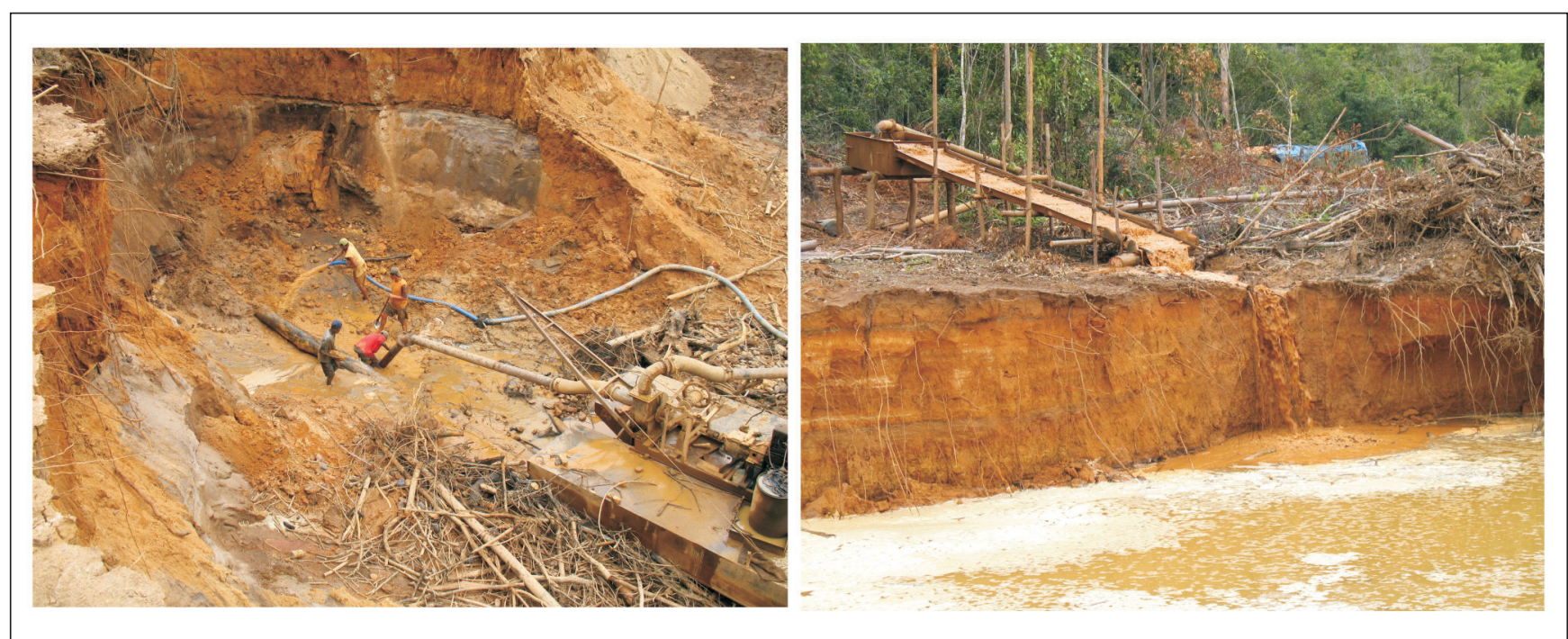

Figura 2 - Processos de desmonte hidráulico do minério e bombeamento (esquerda) deste aos sluices para concentração do ouro em carpetes (direita). Notar descarte do rejeito diretamente no leito do rio. 


\section{Conclusões}

A atividade garimpeira é vista como degradadora do meio ambiente e do recurso mineral, por ser predatória e rudimentar, além de não dispor de um planejamento das operações de extração do minério. As instalações de tratamento/ concentração são pouco eficientes, possibilitando baixa recuperação do bem mineral. O mesmo se faz sentir no Garimpo do Engenho Podre, onde, além dos problemas ambientais inerentes da operação, ocorrem perdas superiores a $40 \%$ na recuperação, segundo estudos efetuados no Departamento de Engenharia de Minas da UFOP.

Segundo Barreto (2001), os principais impactos gerados, na etapa de produção do ouro em garimpos, são: desmatamento de mata ciliar; turbidez, assoreamento dos rios, poluição por mercúrio nos solos, nos sedimentos, nas águas dos rios e no ar, com conseqüências na saúde ocupacional, na biota e na flora. Esses impactos foram verificados no Garimpo do Engenho Podre, embora a poluição por mercúrio tenha sido controlada, após a implantação de um programa de gestão ambiental, que incluiu a construção de um laboratório de amalgamação, queima do amálgama em retorta e disposição controlada do rejeito da amalgamação. O programa de gestão ambiental aplicado ao Garimpo do Engenho Podre constitui uma das etapas em busca da sustentabilidade ambiental da atividade na região.

Embora marginal, do ponto de vista técnico, a atividade garimpeira desenvolve um papel importante como geradora de emprego e aumento de renda, conforme dados econômicos apresentados. Os garimpeiros ligados à COOPERGAMA representam apenas $10 \%$ da população de Monsenhor Horta, mas, por outro lado, a atividade é forte geradora de riquezas para o distrito com grande impacto no comércio local, além do apoio financeiro da cooperativa em projetos sociais e culturais no local.

O desenvolvimento sustentável incorpora estratégias ambientalmente adequadas para promover um desenvolvi- mento sócio-econômico mais eqüitativo. No Garimpo do Engenho Podre, verifica-se que os funcionários envolvidos, mesmo aqueles menos alfabetizados, possuem um poder de compra alto, quando comparados com os dados do quadro nacional, onde aqueles com o mesmo nível de educação ganham apenas o correspondente a um salário mínimo.

Está em andamento um projeto, na FAPEMIG, de transferência de tecnologia, aprovado dentro do Edital de Arranjos Produtivos Locais - APL's de base mineral. Esse projeto está sob a coordenação de professores do Departamento de Engenharia de Minas da Universidade Federal de Ouro Preto e visa, além de montagem de instalações de beneficiamento mais eficientes, ao planejamento de extração do bem mineral e à implantação de um sistema de gestão ambiental.

Paralelamente, o Departamento de Engenharia Florestal da Universidade Federal de Viçosa está envolvido num projeto de revegetação de mata ciliar na área do Garimpo, o qual também obteve apoio financeiro da FAPEMIG. Os projetos da UFOP e, agora, da UFV, em parceria com a COOPERGAMA, demonstram uma mudança de mentalidade dos garimpos em prol de uma atividade mais satisfatória do ponto de vista ambiental e sócio-econômico - ferramentas imprescindíveis para o desenvolvimento sustentável da atividade.

\section{Agradecimentos}

Os autores agradecem a FAPEMIG, pelo financiamento do projeto EDT2526/06 "Agregação de valor tecnológico, funcional e ambiental na extração de ouro por draga no rio Gualaxo do Norte" que possibilitou tal trabalho.

\section{Referências bibliográficas}

BARRETO, M.L. Cierre de Minas: experiências em Iberoamerica. Rio de Janeiro: CYTED/ IMAAC, 2000. p. 225.

BARRETO. Mineração e desenvolvimento sustentável: desafios para o Brasil. Rio de Janeiro: CETEM/MCT, 2001. 225 p.

CLARK, A. L., CLARK, J. C. An integrated methodology for assessing the social and cultural impact of mining. In: Management of Comodity Resources in the Context of Sustainable Development: Social Impact of Mining, Geneva, Switzerland, UNCTAD/ITCD/COM.5. 60-65. 1996.

CRAGG, A. W. Sustainable development and mining: Opportunity or threat to the industry? CIM Bulletin, n. 91, v.1023, p. 45-50, 1998.

EGGERT, R. G. Sustainable development and the mineral industry. In: Sustainable Development and the Future of Mineral Investment. OTTO, J., CORDES, J. (Ed.). Paris, UNEP: 2.1$2.15,2000$.

EPPS, J., BRETT, A. Engaging stakeholders. Sustainable Development and the Future of Mineral Investment. OTTO, J., CORDES, J. (Ed.). Paris, UNEP: 5.1-5.38. 2000.

JAMES, P. M. The triple bottom line: key to project success. Natural Resources. 2000. Information captured on 03/07/2000 at http://www.natural-resources.org/: 1-6.

KHANNA, T. Mine closure and sustainable development. London: Mining Journal Books Ltd., 2000.

MIKESELL, F. M. Viewpoint: sustainable development of mineral resources. Resources Policy, n. 20, v. 2, p. 83, 1994.

MILLER, C. G. Mining and sustainable development: environmental policies and programmes of mining industry associations. UNEP Industry and Environment, n. 20, v. 4, p. 14-17, 1997.

MUNCHENBERG, S. Is there a role for mining in sustainable development? Groundwork, v. 2, n. 2, 1998.

WARHURST, A., M. Macfarlane and G. Wood (1999). Issues in the management of the sócio-economic impacts of mine closure. Environmental Policy in Mining: Coorporate Strategy and Planning for Closure. WARHURST, A., NORONHA, L. London: Lewis Publishers. p.81-100.

Artigo recebido em 15/05/2008 e aprovado em 03/02/2009. 